\title{
Does Salvage Chemotherapy Regimen Intensity Embark on Clearance of Bone Marrow Neuroblastoma?
}

\author{
Mohamed Fawzy ${ }^{1}$, Asmaa Hamoda ${ }^{1}$, Ahmed Elhemaly ${ }^{1}$, Naglaa Elkinaai ${ }^{2}$, Sonya \\ Soliman $^{3}$, Hala Reda ${ }^{3}$, Salma Elmenawi ${ }^{4 *}$, Emad Moussa ${ }^{1,5}$
}

\begin{abstract}
Introduction: Neuroblastoma (NBL) is the most common extracranial solid tumor in children. It accounts for $15 \%$ of the deaths from cancer in the pediatric age group. Approximately half of the newly diagnosed children are at "high risk" (HR) of treatment failure. This study aim was to evaluate the impact of salvage chemotherapy ICE (ifosfamide, carboplatin, and etoposide) versus TC (topotecan/cyclophosphamide) when administered to NBL HR patients having residual bone marrow disease after primary tumor control on the first line treatment regimen. Materials and Methods: The present retrospective study included two groups of eligible stage 4 NBL patients with persistent bone marrow disease. Group (1), 29 patients, received ICE whereas less intensive TC was administered to Group (2), 32 patients. Data analysis included epidemiological variables, pathology subtype, MYCN gene status, primary tumor response and their correlation with bone marrow disease clearance on each regimen. Results: A higher tendency of complete bone marrow clearance was reported in patients who received ICE compared to TC; $41.4 \%$ versus $25.0 \%$, respectively. However, the difference was not statistically significant $(p=0.174)$. Conclusion: TC regimen appears to be a good alternative to ICE as salvage treatment in an attempt to clear NBL bone marrow residual, with the privilege of being less toxic and can be given on outpatient basis. Further randomized trials of larger study sample size with survival impact analysis are warranted.
\end{abstract}

Keywords: Neuroblastoma- refractory bone marrow- ICE- Topotecan/Cyclophosphamide

Asian Pac J Cancer Prev, 20 (5), 1519-1524

\section{Introduction}

Many patients with neuroblastoma (NBL) have metastatic disease at presentation. Approximately half of the newly diagnosed children with NBL are at high risk of treatment failure. The improved characterization of NBL biology has resulted in the possibility of adopting tailored treatment strategies (Maris, 2010). Despite aggressive multimodal therapy, overall survival (OS) for high-risk neuroblastoma (HRNB) is $<50 \%$ at 5 years with most relapses $(80 \%)$ occurring within 2 years of diagnosis (Basta et al., 2016). Bone marrow (BM) is one of the common sites of metastasis, especially in high-risk patients. Induction chemotherapy followed by high dose chemotherapy and stem cell transplantation (SCT) and 13-cis-retinoic acid therapy forms the best current basis of treatment of such cases. It was reported that persistent marrow residual NBL after induction of different treatment strategies and intensities is usually associated with poor outcome (Seeger et al., 2000; Cai et al., 2012). Some studies showed BM remission after nine cycles of induction therapy followed by tandem SCT that remained progression-free (Young et al., 2015). A recent Children's oncology group meta-analysis showed a 4 -year progression-free survival of $6 \%$ and OS of $15 \%$ for high-risk patients enrolled on early phase trials for relapsed/refractory disease (London et al., 2017).

The significance of persistent bone marrow metastatic disease and its impact on survival as a probable risk factor remained questionable. However, previous studies reported an improved outcome of HR-NB with prolonged induction and intensified therapy (Sung et al., 2007). Unlike expected, the degree of volume reduction during early induction higher in tumors with amplified MYC-N gene and undifferentiated histology, higher Neuron-specific enolase (NSE), lower urinary Vanillyl mandelic acid (VMA) and at younger age patients (Yoo et al., 2013; Young et al., 2015).

Topotecan and cyclophosphamide are both active drugs in neuroblastoma, and various combinations of these two drugs with or without other additional drugs have been used in a number of phase II trials. There are no FDA(food and drug administration) approved drugs for neuroblastoma, and therefore this regimen

${ }^{1}$ Department of Pediatric Oncology, ${ }^{2}$ Department of Pathology, ${ }^{3}$ Department of Clinical Pathology, ${ }^{4}$ Clinical Research Uni, Research Department, Children Cancer Hospital Egypt-57357 and National Cancer Institute (NCI), Cairo University, ${ }^{5}$ Menoufeya Faculty of Medicine, Cairo,Egypt.*For Correspondence: s.menawi@gmail.com 
and the individual drugs are considered off-label and experimental, but it is medically necessary based on phase II studies, one phase III study and the lack of alternatives. Phase III studies are challenging to perform in this disease because of the extensive use of bone marrow transplantation (Saylors et al., 2001)

Topotecan was primarily used in combination with cyclophosphamide, and phase II study using cycles of this combination $\left(250 \mathrm{mg} / \mathrm{m}^{2} /\right.$ day cyclophosphamide and 0.75 $\mathrm{mg} / \mathrm{m}^{2} /$ day topotecan for 5 days each) achieved objective responses in 6 of 13 neuroblastoma patients, other studies showed increased response rates in patients treated with the cyclophosphamide/topotecan combination compared to topotecan alone ( $2 \mathrm{mg} / \mathrm{m}^{2} /$ day for 5 days), although no difference in OS rates were observed (Sarah B. Whittle et al., 2017).

Topotecan targets the DNA-relaxing enzyme topoisomerase I, resulting in covalent binding of the enzyme to DNA, in turn causing lethal DNA damage during replication (Takimoto et al., 2001).

\section{Materials and Methods}

\section{Patients, Treatment, and Evaluation}

The study included HR NBL patients (based on COG staging system) from 0-18 years of age at presentation to Children Cancer Hospital Egypt 57357 (CCHE) during the period from July 2007 till July 2016.

Enrolled patients had refractory bone marrow disease after induction therapy with COG A3973 or SFOP NBL90-derived induction regimen (cyclophosphamide, vincristine, and doxorubicin alternating with etoposide and carboplatin) (Kreissman et al., 2013; Bergeron et al., 2005).

\section{Two groups of eligible stage 4 NBL patients were identified}

ICE group consisted of 29 patients receiving ICE on an inpatient basis, whereas the less intensive TC was administered to another group of 32 patients on an outpatient basis.

Salvage chemotherapy ICE consisted of (Ifosfamide 1.8 $\mathrm{g} / \mathrm{m} 2$ day $1-5$, Carboplatin $450 \mathrm{mg} / \mathrm{m} 2$ day 1 and Etoposide $100 \mathrm{mg} / \mathrm{m} 2$ day $1-5)$, while TC consisted of intravenous Topotecan $0.75 \mathrm{mg} / \mathrm{m} 2 /$ day and cyclophosphamide 250 $\mathrm{mg} / \mathrm{m} 2 /$ day delivered for 5 days, both salvage regimens were given every 21 days. Weight-based dosing was used for children under $12 \mathrm{~kg}$.

Post-induction bone marrow evaluation has been done. Evaluation of primary and distant sites, other than bone marrow, by CT (Computed Tomography) or MRI (Magnetic resonance imaging), was done to exclude progression.

Patients, who achieved bone marrow remission, continued on the same salvage line until a bone marrow transplant (BMT).

Those with refractory bone marrow after three cycles or progressive patients at any point of salvage treatment were considered off-protocol.

\section{Statistical methods}

Medical records were used for retrieving data that were eligible for analysis.

Data management was done using Redcap (Research Electronic Data Capture) tool hosted at CCHE.

Quantitative data were expressed as median and range, and qualitative data were expressed as frequency and percentage.

Mann-Whitney U test was used for comparison of continuous variables between both groups, while Pearson's chi-squared test was used for comparison of frequencies between groups. Kaplan Meier estimated overall survival (OS); the difference in OS was compared using the log-rank test. For OS, time to event was defined as the time from enrollment on protocol till death due to any cause or till the date of the last contact in case of no event. Survival estimate is reported as 3-year survival, and the related $95 \%$ CIs were calculated.

The previous statistical calculations were done using SPSS (Statistical Package for the Social Science; IBM, USA) version 20 for Microsoft Windows.

To estimate progression incidence (PI) and non-disease related Mortality (NDM), cumulative incidence functions were used; progression and death from non-disease related causes were considered competing for events. Gray's method was used to estimate the cumulative incidence of PI and NDM and the equality between groups based on the regimen, to compare the cumulative incidence of a specific type of failure across groups.

R package 'cmprsk' for sub-distribution Analysis of Competing Risks was used (Robert and Gray 1988).

A probability value (p-value) less than 0.05 was considered statistically significant.

\section{Results}

This retrospective study included 61 HR NBL patients with refractory bone marrow disease at end of induction with slight male predominance $\mathrm{M}: \mathrm{F}=2: 1$, nearly all the study group were more than 1.5 years old with the exception of 1 patient who was less than $1.5 \mathrm{y}$, unfavorable histopathology was predominant in (46/61) patients (75.4\%) however MYC-N amplification was present only in $8 / 61$ patients $(13.1 \%)$ with the majority having adrenal gland as primary tumor site. At the start of operation in our hospital we used the SFOP 90 protocol from 2007 till 2012 then we shifted to new COG protocol A3973 (which is much more intensified) from 2012 till now; in ICE arm (29 patients ) (17/29)patients received less intensity induction (SFOP) compared to $12 / 29$ patients who received A3973 protocol.

Out of 61 patients, 29 received ICE and 32 received TC. Patients' characteristics in each group are shown in Table 1.

Regarding bone marrow clearance, 20/61 (32.8\%) achieved bone marrow clearance on both regimens, while $41 / 61(67.2 \%)$ patients failed to obtain bone marrow remission.

In the ICE group, $12 / 29$ patients (41.4\%) achieved bone marrow remission, while only $8 / 32$ TC patients (25\%) reached clear bone marrow. Comparing bone marrow remission in both groups showed no significant difference $(\mathrm{p}=0.174)$ (Table 2$)$. 
Table 1. Patients' Characteristics in Each Group

\begin{tabular}{|c|c|c|}
\hline & \multicolumn{2}{|c|}{ Salvage regimen } \\
\hline & ICE & $\mathrm{TC}$ \\
\hline \multicolumn{3}{|l|}{ OS status } \\
\hline Alive & $9(31 \%)$ & $19(59 \%)$ \\
\hline Dead & $20(69 \%)$ & $13(41 \%)$ \\
\hline \multicolumn{3}{|l|}{ Age category } \\
\hline $365 \mathrm{~d}$ to less than $547 \mathrm{~d}$ & 0 & $1(3 \%)$ \\
\hline 547 and above & $29(100 \%)$ & $31(97 \%)$ \\
\hline \multicolumn{3}{|l|}{ Pathology } \\
\hline Ganglioneuroblastoma & $4(14 \%)$ & $3(9.4 \%)$ \\
\hline Neuroblastoma & $25(86 \%)$ & $29(90.6 \%)$ \\
\hline \multicolumn{3}{|l|}{ Shimada } \\
\hline FH & $3(10 \%)$ & $2(6.2 \%)$ \\
\hline UH & $19(66 \%)$ & $27(84.4 \%)$ \\
\hline NA & $7(24 \%)$ & $3(9.4 \%)$ \\
\hline MYCN & $4(14 \%)$ & $4(12 \%)$ \\
\hline Not amplified & $13(45 \%)$ & $22(69 \%)$ \\
\hline NA & $12(41 \%)$ & $6(19 \%)$ \\
\hline \multicolumn{3}{|l|}{ 1ry site } \\
\hline Abdominal (nonadrenal) & $1(3.4 \%)$ & $2(6.2 \%)$ \\
\hline adrenal & $21(72.4 \%)$ & $28(87.6 \%)$ \\
\hline thoracic & $7(24.2 \%)$ & $2(6.2 \%)$ \\
\hline \multicolumn{3}{|l|}{ BMT done after salvage } \\
\hline No & $21(72 \%)$ & $28(87.5 \%)$ \\
\hline Yes & $8(28 \%)$ & $4(12.5 \%)$ \\
\hline \multicolumn{3}{|l|}{ Induction regimen } \\
\hline A3973* & $12(41 \%)$ & $32(100 \%)$ \\
\hline SFOP NBL90\# & $17(59 \%)$ & 0 \\
\hline Total & 29 & 32 \\
\hline
\end{tabular}

Does Salvage Regimen Embark on Neuroblastoma Marrow

Duration of bone marrow (BM) remission for ICE patients ranged between 1.44 and 56.7 months with median BM remission time of 7.8 months, while, in $\mathrm{T} / \mathrm{C}$ patients, it ranged between 0.52 and 17.52 months with a median time 12.46 months $(\mathrm{p}=0.643)$ (Figure 1).

For the whole patient cohort, 3-year OS was 56.1\% (95\% CI: 43.36-68.84); 3-year OS of ICE and TC groups were $51.1 \%$ (95\% CI: 32.68-69.52) and 60.2\% (95\% CI: 42.36-78.04) $(\mathrm{p}=0.120)$, respectively (Figure 2).

Progression incidence of the whole group was 54.5\% (95\% CI: 40.4-66.5), as shown in Figure 3. When comparing Progression incidence of both groups, Progression incidence of ICE patients was 55.2\% (95\% CI: 35-71.4) versus 53.6\% (CI: 33.2-70.2) in T/C patients, which was statistically insignificant $(\mathrm{p}=0.5)$. Median time to progression (TTP) for all study patients was 32 months. While the TTP of ICE group was 24 months, TTP for TC group was 34.62 months (Figure 4).

Out of the 29 patients who took ICE, 8 (28\%) had sustained bone marrow remission allowing them to undergo BMT; as for the $31 \mathrm{~T} / \mathrm{C}$ patients, only 4 (12.5\%) had sustained bone marrow remission and had BMT.

\section{Discussion}

Around $50 \%$ of children with HR-NBL will be refractory to therapy or suffer a relapse, both of which are invariably fatal. Bone marrow was reported to be by far one of two most frequent sites altogether with bone showing refractoriness in such patients (Matthay et al., 1993; DuBois et al., 1999).

Histologically persistent BM tumor was associated with worse prognosis even after tandem HDCT/auto-SCT (Young et al., 2015). Some investigators found that persistent BM involvement before consolidation therapy was an independent adverse prognostic factor (Mora et al., 2015). The same relation was reported by the European

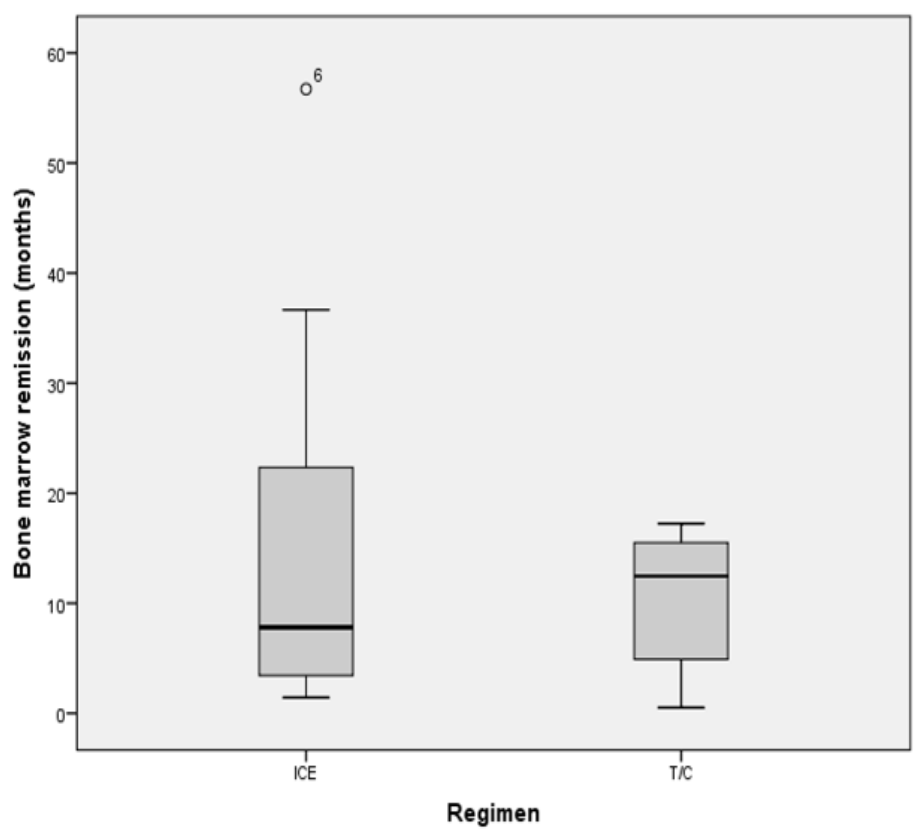

Figure 1. Box Plot Showing Distribution of Bone Marrow Remission Values of the Two Groups. T/C, Topotecan/ Cyclophosphamide; ICE, Ifosfamide/Carboplatin/Etoposide 
Table 2. Impact of Different Salvage Regimens on Bone Marrow Clearance

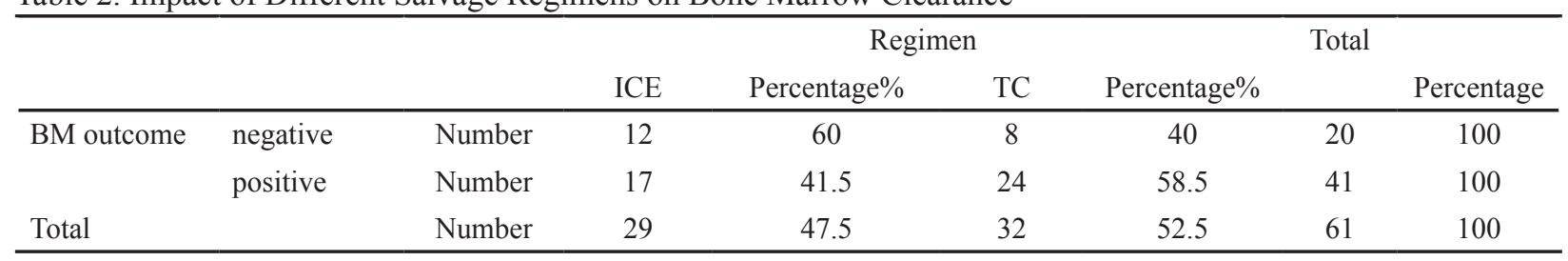

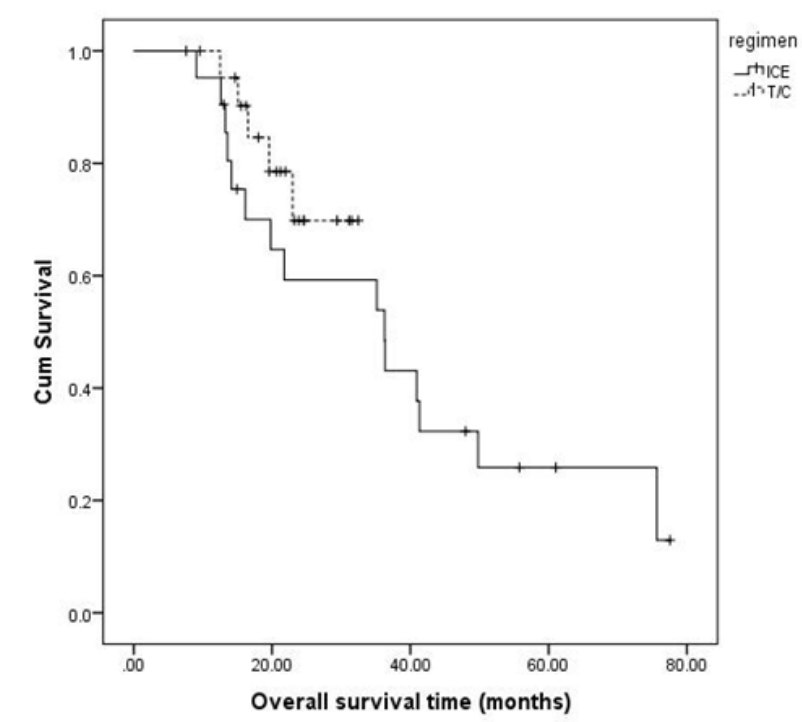

Figure 2. Three -Year Overall Survival of Both Salvage Regiemens. Note that T/C shows better 3-year overall survival than ICE with no significant difference, $\mathrm{p}=0.12 ; \mathrm{T} / \mathrm{C}$, topotecan/cyclophosphamide; ICE, ifosfamide/carboplatin/ etoposide.

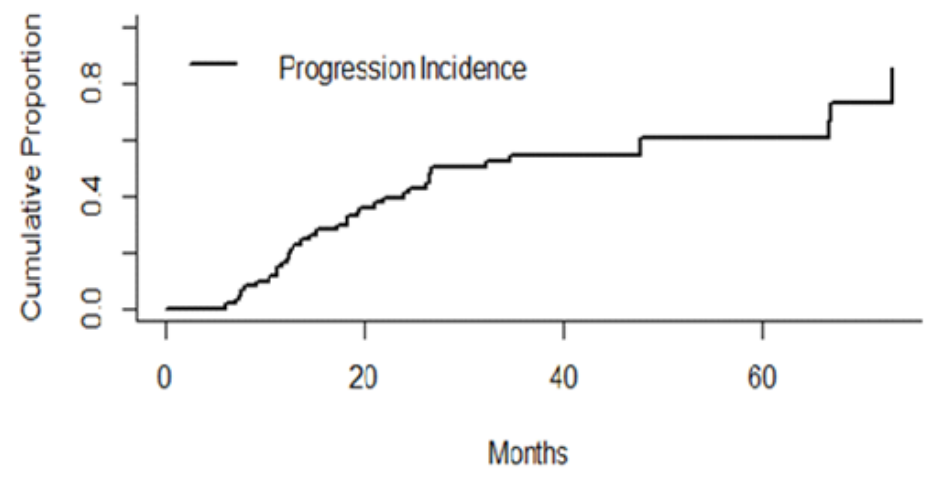

Figure 3. Three-Year Progression Incidence of the Whole Study Cohort

Bone Marrow Transplant (EBMT) registry analysis (Ladenstein et al., 1998; Seeger et al., 2000; Fukuda et al., 2001; Cai et al., 2012).
On the other hand, the good prognostic value of rapid BM molecular response in NBL was confirmed by others (Stutterheim et al., 2011).

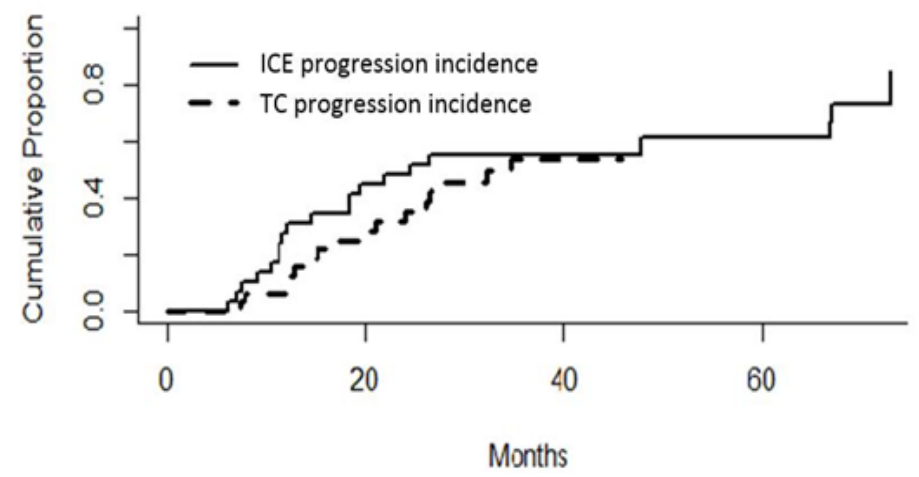

Figure 4. Three -Year Progression Incidence by Regimen. Progression incidence of ICE patients was slightly higher than T/C patients, $\mathrm{p}=0.5$. T/C, topotecan/cyclophosphamide; ICE, ifosfamide/carboplatin/etoposide 
Highly tumorigenic tumor spheres were isolated from the bone marrow of patients with HR NB suggested that BM clearance is usually delayed in NBL patients (Hansford et al., 2007).

Acquired alkylator resistance likely contributes to refractory BM disease. In a pilot study, rapid maximal use of chemotherapy and surgery together with an adapted to response cycles of high-dose topotecan and cyclophosphamide ( $\mathrm{mN} 7$ protocol) rendered two-thirds of high-risk stage 4 NBL patients into $\mathrm{CR}$. The combination of topotecan and cyclophosphamide was attractive because of non-overlapping toxicities and the significant therapeutic synergy demonstrated. Topo/Cyclo proved to be superior to topotecan alone in terms of PFS, yet with no impact on OS (London et al., 2010; Mora et al., 2015). Other studies reported response to ICE used as salvage therapy in 50\% with refractory NBL and in 35\% for disease progression during chemotherapy $(\mathrm{P}=0.005)$ (Kushner et al., 2013).

Higher doses of cyclophosphamide and topotecan with vincristine have also shown an overall response rate of $19 \%$ for patients with primary refractory neuroblastoma and $52 \%$ for those with the first relapse

In the present study, residual BM infiltration was seen after standard induction therapy in 61 study cases of HR-NBL. Two different post-induction salvage regimens (conventional ICE versus Topo/Cyclo) were alternatively used on a randomized basis; aiming to achieve complete $\mathrm{BM}$ remission prior to autologous stem cell transplant. Bone marrow remission rate was achieved in $41.4 \%$ after ICE regimen versus $25 \%$ after Topo/Cyclo $(p=0.174)$.

On the other hand, the median duration of maintained BM clearance was 12.46 months after Topo/Cyclo with higher 3 year- OS than $60.2 \%$ versus 7.8 months only after ICE and $51.1 \%$ OS; meaning that Topo/Cyclo can result in longer sustained remission and higher OS than ICE; however none was statistically proven ( $p=0.64$ and $\mathrm{p}=0.12$, respectively).

Besides, the median time to progression (TTP) (time at which $50 \%$ of the patients had progressed) for whole study patients was 32 months; 34.62 months for topo/ Cyclo versus 24 months for ICE subsets.

Unlike ICE, more convenience to Topo/Cyclo regimen administered on an outpatient basis with much lesser need for post cycle admission and supportive care favoring a better quality of life was noticed in this study.

Although untreated neuroblastoma have a relative paucity of mutations in therapeutically target genes, relapsed neuroblastoma have been found to have a number of mutations, clinical trials based on genetic mutation identified in relapsing tumors have demonstrated both feasibility and efficacy, suggesting that molecularly targeted therapy may lead to increased rates of response and improved outcomes for children with relapsed and refractory neuroblastoma (Eleveld et al., 2015; Padovan-Merhar et al., 2016).

Results of the present study did not reveal the superiority of any of the two salvage regimens used on the outcome of patients with HR-NBL having residual post-induction BM disease. Despite the lack of statistical significance of the relations tested between each patients'
Does Salvage Regimen Embark on Neuroblastoma Marrow

subset outcome and different study variables yet, preliminary data referred to numerically longer sustained BM remission, higher OS and longer TTP with Topo/Cyclo in comparison to ICE. Further data analysis on a larger study patients' sample to reach a solid ground conclusion is recommended.

\section{Acknowledgments}

This study was not funded by any organization and was approved by our hospital' scientific and ethical committee; the authors declared that they did not have any conflict of interest.

\section{References}

Basta NO, Halliday GC, Makin G, et al (2016). Factors associated with recurrence and survival length following a relapse in patients with neuroblastoma. Br J Cancer, 115, 1048 e57.

Bergeron C, Dubourg L, Chastagner P, et al (2005). Long-term renal and hearing toxicity of carboplatin in infants treated for localized and unresectable neuroblastoma: Results of the SFOP NBL90 study. Pediatr Blood Cancer, 45, 32-6.

Cai JY, Pan C, Tang YJ, et al (2012). Minimal residual disease is a prognostic marker for neuroblastoma with bone marrow infiltration. Am J Clin Oncol, 35, 275-8.

DuBois SG, Kalika Y, Lukens JN, et al (1999). Metastatic sites in stage IV and IVS neuroblastoma correlate with age, tumor biology, and survival. J Pediatr Hematol Oncol, 21, 181-9.

Eleveld TF, Oldridge DA, Bernard V, et al (2015). Relapsed neuroblastomas show frequent RAS-MAPK pathway mutations. Nat Genet, 47, 864-71.

Fukuda M, Miyajima Y, Miyashita Y, et al (2001). Disease outcome may be predicted by molecular detection of minimal residual disease in bone marrow in advanced neuroblastoma: a pilot study. J Pediatr Hematol Oncol, 23, 10-3.

Hansford LM, McKee AE, Zhang L, et al (2007). Neuroblastoma Cells isolated from bone marrow metastases contain a naturally enriched tumor-initiating cell. Cancer Res, 67, 23.

Kreissman SG, Seeger RC, Matthay KK, et al (2013). Purged versus non-purged peripheral blood stem-cell transplantation for high-risk neuroblastoma (COG A3973): a randomized phase 3 trial. Lancet Oncol, 14, 999-1008.

Kushner BH, Modak S, Kramer K, et al (2013). Ifosfamide, carboplatin, and etoposide for neuroblastoma: a high-dose salvage regimen and review of the literature. Cancer, 119, 665-71.

Lacayo NJ (2017). Pediatric NBL Treatment and Management. Medscape [Online]. Available at: https://emedicine. medscape.com/article/988284-treatment\#d5b. Accessed August 13, 2018.

Ladenstein R, Philip T, Lasset C, et al (1998). Multivariate analysis of risk factors in stage 4 neuroblastoma patients over the age of one year treated with megatherapy and stem-cell transplantation: a report from the European Bone Marrow Transplantation Solid Tumor Registry. J Clin Oncol, 16, 953-65.

London WB, Frantz CN, Campbell LA, et al (2010). Phase II randomized comparison of topotecan plus cyclophosphamide versus topotecan alone in children with recurrent or refractory neuroblastoma: A children's oncology group study. J Clin Oncol, 28, 3808-15.

London WB, Bagatell R, Weigel BJ, et al (2017). Historical time to disease progression and progression-free survival in patients with recurrent/refractory neuroblastoma treated in 
the modern era on Children's Oncology Group early-phase trials. Cancer, 123, 4914e23

Maris JM (2010). Recent advances in neuroblastoma. $N$ Engl $J$ Med, 362, 2202-11.

Matthay KK, Atkinson JB, Stram DO, et al (1993). Patterns of relapse after autologous purged bone marrow transplantation for neuroblastoma: a Children's Cancer Group pilot study. J Clin Oncol, 11, 2226-33.

Mora J, Cruz O, Lavarino C, et al (2015). Results of induction chemotherapy in children older than 18 months with stage- 4 neuroblastoma treated with an adaptive-to-response modified N7 protocol (mN7). Clin Transl Oncol, 17, 521-9.

Padovan-Merhar OM, Raman P, Ostrovnaya I, et al (2016). Enrichment of targetable mutations in the relapsed neuroblastoma genome. PLoS Genet, 12, e1006501.

Robert J, Gray A (1988). Class of K -sample tests for comparing the cumulative incidence of a competing risk. Ann Stat, 16, 1141-54.

Sarah B Whittle, Valeria S, Erin D, et al (2017) Overview and recent advances in the treatment of neuroblastoma. Expert Rev Anticancer Ther, 17, 369-86.

Sailors Robert L, Stine Kimo C, Sullivan J, et al (2001) Cyclophosphamide plus topotecan in children with recurrent or refractory solid tumors: A pediatric oncology group phase II study. J Clin Oncol, 19, 3463-9.

Seeger RC, Reynolds CP, Gallego R, et al (2000). Quantitative tumor cell content of bone marrow and blood as a predictive of outcome in a stage IV Neuroblastoma: a Children's Cancer Group Study. J Clin Oncol, 18, 4067-76.

Stutterheim J, Zappeij-Kannegieter L, Versteeg R, et al (2011). The prognostic value of fast molecular response of marrow disease in patients aged over one year with stage 4 neuroblastoma. Eur J Cancer, 47, 1193-202.

Sung KW, Lee SH, Yoo KH, et al (2007). Tandem high dose chemotherapy and autologous stem cell rescue in patients over one year of age with stage 4 neuroblastoma. Bone Marrow Transplant, 40, 37-45.

Takimoto CH, Arbuck SG (2001). Topoisomerase I targeting agents: The camptothecins, in Chabner BA, Longo DL (eds): Cancer Chemotherapy and Biotherapy. Philadelphia, PA, Lippincott Williams and Wilkins, 2001, pp 579-604.

Yoo SY, Kim JS, Sung KW, et al (2013). The degree of tumor volume reduction during the early phase of induction chemotherapy is an independent prognostic factor in patients with high-risk neuroblastoma. Cancer, 119, 656-64.

Young BC, Go EB, Na HL, et al (2015). Clinical significance of persistent tumor in bone marrow during treatment of high-risk neuroblastoma. J Korean Med Sci, 30, 1062-7.

This work is licensed under a Creative Commons AttributionNon Commercial 4.0 International License. 